\title{
Cultural characteristics of Cordyceps militaris strain 'Yedang 3' on various media and nutritional conditions
}

\author{
Byung-Joo Lee ${ }^{1 *}$, Mi-Ae Lee ${ }^{1}$, Yong-Gyun Kim ${ }^{1}$, Kwang-Won Lee ${ }^{1}$, \\ Young-Sang Choi ${ }^{2}$, Byung-Eui Lee ${ }^{3}$ and Ho-Yeon Song ${ }^{4}$ \\ ${ }^{1}$ Crop Research Division, Chungcheongnam-do Agricultural Research \& Extension Services, Yesan 340-861, Korea \\ ${ }^{2}$ Yedang Mushroom Institute, Yesan 305-764, Korea \\ ${ }^{3}$ Industry Academy Cooperation Foundation, Soonchunghyang University, Asan 336-745, Korea \\ ${ }^{4}$ College of Medicine, Soonchunghyang University, Chunan 330-930, Korea
}

(Received September 13, 2013 / Revised September 21, 2013 / Accepted September 27, 2013)

\begin{abstract}
The effects of environmental and nutritional requirement such as temperature, pH, different media, carbon, nitrogen, and carbon-to-nitrogen ratio on the mycelial growth of Cordyceps militaris strain 'Yedang 3' were studied. The optimum temperature and $\mathrm{pH}$ for the growth of mycelium were $20-25^{\circ} \mathrm{C}$ and $\mathrm{pH}$ 6-7, respectively. Out of ten media tested, mushroom complete media (MCM) was the best medium for fast mycelial growth, and Sabouraud's dextrose agar yeast extract (SDAY), malt extract yeast extract agar (YMA) also were favored. The color and shape of colonies varied in different media. The best carbon sources for mycelial growth were fructose, mannitol, and sucrose, whereas the best nitrogen sources were tryptone and peptone. However, mycelia grew slowly in inorganic nitrogen compounds such as $\mathrm{NH}_{4} \mathrm{Cl},\left(\mathrm{NH}_{4}\right)_{2} \mathrm{SO}_{4}, \mathrm{NH}_{4} \mathrm{NO}_{3}$, and $\mathrm{NaNO}_{3}$. The optimum $\mathrm{C}$ : $\mathrm{N}$ ratio observed on the culture media was 30-40 range. These results provided basic information on cultural characteristics of vegetative growth and might be useful for spawn production in Cordyceps militaris.
\end{abstract}

KEYWORDS - Carbon-to-nitrogen ratio, Cordyceps militaris, Culture medium, Mycelial growth, Temperature

\section{Introduction}

Mushrooms have been sought and cultivated for the production of food consumption due to its nutritive and medicinal values (Fan et al., 2006). Cordyceps species are important mushrooms traditionally used for healths and vitality. C. sinensis (syn. Ophiocordyceps sinensis) is the most highly sought-after species among them and its main bioactive metabolite is cordycepin (3'-deoxyadenosine). Cordycepin has been found to be pharmacologically functional such as anti-tumor, immunomodulation, anti-inflammatory, anti-virus, anti-leukemic, anticancer, anti-diabetic, preventing and treating of obesity (De Julian-Ortiz et al., 1999; Masuda et al., 2007; Wong et al., 2010; Wu et al., 2011; Yoo et al., 2004). However, artificial production of fruiting bodies in $C$. sinensis has not yet to be achieved (Guo and Yang, 1999; Jiang and Yao, 2003; Liu et al., 1999).
It was found that most of active constituent of $C$. militaris was similar to those of $C$. sinensis including cordycepin and even would be better in some medicinal compounds (Das et al., 2010; Oh et al., 2003; Patel and Ingalhalli, 2013; Yu et al., 2006; Zhou et al, 2009). C. militaris can be cultivated artificially and its natural compounds are similar to artificially cultured ones (Jiang and Sun, 1999; Tong et al., 1997). Therefore, it has been considered as a alternative to $C$. sinensis for a wide variety of purposes (Dong et al., 2012; Gao, 2008; Gui and Zhu, 2008).

C. militaris is an entomopathogenic fungus infecting mainly on Lepidoptera larvae. It belongings to the genus Cordyceps in family Clavicipitaceae of order Hypocreales, class Ascomycetes. The genus Cordyceps has a worldwide distribution from China, Japan, Britain, Norway, Nepal and Korea comprising of approximately 400 species (Liang, 2001; Liu, 1999; Ma et al., 2007; Stensrud et al., 2005; Sung et al.,

*Corresponding author: byungjoo@korea.kr 
2007; Wang et al., 2008). They are particularly abundant and diverse in humid temperate and tropical forests.

The total genome of C. militaris was $32.2 \mathrm{Mb}$, which is composed of seven chromosomes ranging between 2.0 and $5.7 \mathrm{Mb}$ (Wang et al., 2010; Zheng et al., 2011). In the sexual reproduction, $C$. militaris is dominantly heterothallic having a single locus with two alternate allelic mating system mediated by mating-type (MAT) genes (Wen et al., 2012; Yokoyama et al., 2003; 2004; Zheng et al., 2011). There are two types of MAT gene: MAT1-1-1 which is characterized by the presence of DNA sequence encoding an alpha-domain protein and MAT1-2-1 which is characterized by the presence of sequence encoding a high mobility group domain protein (Tan et al., 2011; Turgeon and Yoder 2000). When two different mating-types are present, it can be compatible to generate perithecial fruiting bodies which have both MAT1-1-1 and MAT1-2-1 (Li, 2007).

New strains were bred by crossing single spore strains (Choi et al., 2009; Du et al., 2010; Sun et al., 2009) and UV induced mutagenesis (Che et al., 2004). Cultivation of C. militaris mycelium using artificial media has been developed by different methods expecially for high cordycepin production (Hung et al., 2009; Mao and Zhong, 2006) and the fruiting body has also been produced artificially on various media (Choi et al., 1999; Das et al., 2010; Dong et al., 2012; Sato and Shimazu, 2002; Sung et al., 2002). However, the fruiting body production is unstable and variable in serial subcultures of the same strain compared to the initial cultivation, which was mainly caused by degeneration of the strains (Sung et al., 2006; Wen et al., 2012).

This study was carried out to investigate the effect of basic environment and nutritional conditions such as temperature, $\mathrm{pH}$, carbon source and nitrogen source affecting on the mycelia growth of $C$. militaris strain 'Yedang 3'. This could help to understand the cultural characteristic of mycelial growth and further cultivation of fruiting bodies in commercial production.

\section{Materials and Methods}

\section{Fungul strain}

C. militaris 'Yedang 3' was originally bred by crossing single ascospore isolates C0601S05 and C0601S33 in Yesan, Chungnam, Korea in 2006. It produced more fruiting bodies and contained more cordycepin content (Choi et al., 2009). It's colonies were maintained and subcultured individually onto fresh PDA plates. Then each mycelium was determined in the colony diameter and morphology type of each treatment.

\section{pH, temperature and light}

For $\mathrm{pH}$ experiment of $C$. militaris 'Yedang 3' was studied by growing the mycelial cultures on PDA with a $\mathrm{pH}$ range of $3,4,5,6,7,8,9$ adjusted by $1 \mathrm{~mol} / \mathrm{l}$ hydrochloric acid or $1 \mathrm{~mol} / \mathrm{l}$ sodium hydroxide solution. The effect of temperature on mycelial growth was studied at 5, 10, 15, 20, 25 and $30^{\circ} \mathrm{C}$ in a growth chamber. The experiment was performed in triplicate. Mycelial growth on each plate was measured at 3 directions and average value was calculated with those 3 measurements. All of the inoculated culture media were tested either in the dark or light at $24^{\circ} \mathrm{C}$. Fluorescent lamps were used for light source at $14.3 \mathrm{Wm}^{-2}$ in an illumination incubator.

\section{Mycelial culture and media}

Mycelia of $C$. militaris strains were cultured vegetatively at $24^{\circ} \mathrm{C}$ on PDA medium. The medium was sterilized at $121^{\circ} \mathrm{C}$ for 20 minutes. Agar blocks of $5 \mathrm{~mm}$ in diameter were taken from actively growing colonies on PDA plates and inoculated into each culture media for mycelial growth (Table 1). The experiment was placed by completely randomized design with 3 replications and performed twice.

\section{Carbon and nitrogen sources}

As the basal medium $2 \%$ water agar was used and it was supplemented with twelve different carbon and twelve different nitrogen sources at $2 \%(\mathrm{w} / \mathrm{v})$ concentration. Carbon sources used were 
Table 1. Culture media and their constituents used for mycelial growth

\begin{tabular}{|c|c|c|c|c|c|c|c|c|c|c|}
\hline \multirow{2}{*}{ Composition } & \multicolumn{10}{|c|}{$\operatorname{Media}^{a}(g / l)$} \\
\hline & GT & HAM & HEN & HOP & $\mathrm{MC}$ & MCM & MYA & PDA & SDAY & YMA \\
\hline Agar & 20 & 20 & 20 & 20 & 20 & 20 & 20 & 20 & 15 & 20 \\
\hline \multicolumn{11}{|l|}{ Asparagine } \\
\hline Dextrose & & 10 & & & & 20 & 4 & 20 & 40 & \\
\hline Ebiose & & 5 & & & & & & & & \\
\hline Hyponex & & 3 & & & & & & & & \\
\hline Glucose & 5 & & 50 & 10 & & & & & & 4 \\
\hline Malt-extract & & & & & 20 & & 10 & & & 10 \\
\hline \multicolumn{11}{|l|}{ Maltose } \\
\hline Peptone & & & & & 2 & 2 & & & 10 & \\
\hline Potato & & & & & & & & 200 & & \\
\hline \multicolumn{11}{|l|}{ Sucrose } \\
\hline Tryptone & 10 & & & & & & & & & \\
\hline Yeast-extract & 3 & 3 & & & 2 & 2 & 4 & & 10 & 4 \\
\hline $\mathrm{NaNO}_{3}$ & & & 2 & & & & & & & \\
\hline $\mathrm{K}_{2} \mathrm{HPO}_{4}$ & & & & & 1 & 1 & & & & \\
\hline $\mathrm{MgSO}_{4}$ & & & 0.5 & 0.5 & 0.5 & 0.5 & & & & \\
\hline \multicolumn{11}{|l|}{$\mathrm{KCl}$} \\
\hline \multicolumn{11}{|l|}{$\mathrm{FeSO}_{4}$} \\
\hline $\mathrm{CaCl}_{2}$ & & & 0.1 & & & & & & & \\
\hline $\mathrm{KH}_{2} \mathrm{PO}_{4}$ & & & 1 & 0.1 & 0.5 & 0.46 & & & & \\
\hline $\mathrm{KNO}_{3}$ & & & 2 & 2 & & & & & & \\
\hline
\end{tabular}

a'GT, glucose trypton; HAM, Hamada; HEN, Hennerberg; HOP, Hoppkins; MC, mushroom complete; MCM, mushroom complete media; MYA, malt extract yeast extract agar; PDA, potato dextrose agar; SDAY, Sabouraud's dextrose agar yeast extract; YM, yeast malt; YMA, yeast extract malt extract agar.

fructose $(\mathrm{C} 1)$, galactose $(\mathrm{C} 2)$, glucose $(\mathrm{C} 3)$, mannitol (C4), dextrose (C5), lactose (C6), maltose (C7), sucrose (C8), sorbitol (C9), dextrin (C10), xylose (C11), starch (C12). Nitrogen sources were ammonium phosphate (N1), calcium nitrate (N2), glycine $(\mathrm{N} 3)$, potassium nitrate $(\mathrm{N} 4)$, yeast extract (N5), malt extract (N6), peptone (N7), typtone (N8), ammonium chloride (N9), ammonium sulfate (N10), sodium nitrate (N11), ammonium nitrate (N12). The basal medium was adjusted to $\mathrm{pH} 6$ and sterilized for $20 \mathrm{~min}$ at $121^{\circ} \mathrm{C}$, and aseptically added into a plate at $2 \%(\mathrm{w} / \mathrm{v})$. A $5 \mathrm{~mm}$ diameter plug of inoculum was taken from PDA plate, placed in the center of basal media containing one of carbon and nitrogen sources and incubated at $24^{\circ} \mathrm{C}$. The carbonto-nitrogen $(\mathrm{C}: \mathrm{N})$ ratio was adjusted to 10:1, 20:1, $30: 1$, and 40:1 with varying quantities of sucrose as carbon source and $5 \mathrm{~g} / \mathrm{l}$ of peptone as sole nitrogen source. The carbon content of sucrose was calculated based on the molecular weight of $\mathrm{C}_{12} \mathrm{H}_{22} \mathrm{O}_{11}$ as $42 \%$ and the nitrogen content of the peptone was determined based on the analysis of Kjedalhl method as $12 \%$.

\section{Result and Discussion}

The $\mathrm{pH}$ value for a favorable mycelial growth of C. militaris was in the range of $\mathrm{pH}$ 6 7 (Fig. 1). With regard to the low $\mathrm{pH}$ at 3 to 4 , the fungus grew very slowly with an average growth rate of less than $1.5 \mathrm{~mm} /$ day. Sung et al. (2002) and Park et al. (2001) reported that mycelia growth of $C$. militaris was optimal at $\mathrm{pH} 6$ in agreement with this study but the mycelia grew slowly in 'Yedang 


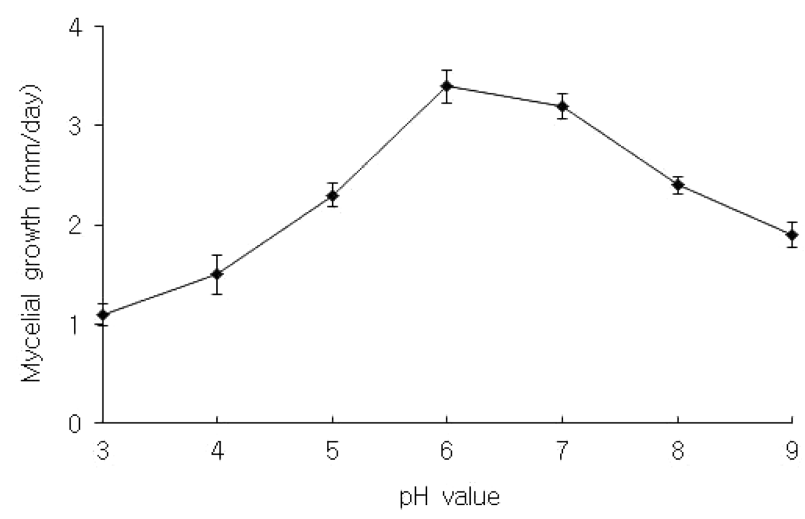

Fig. 1. Mycelial growth of $C$. militaris 'Yedang 3' on the PDA at different $\mathrm{pH}$ value. Bars indicate standard errors.

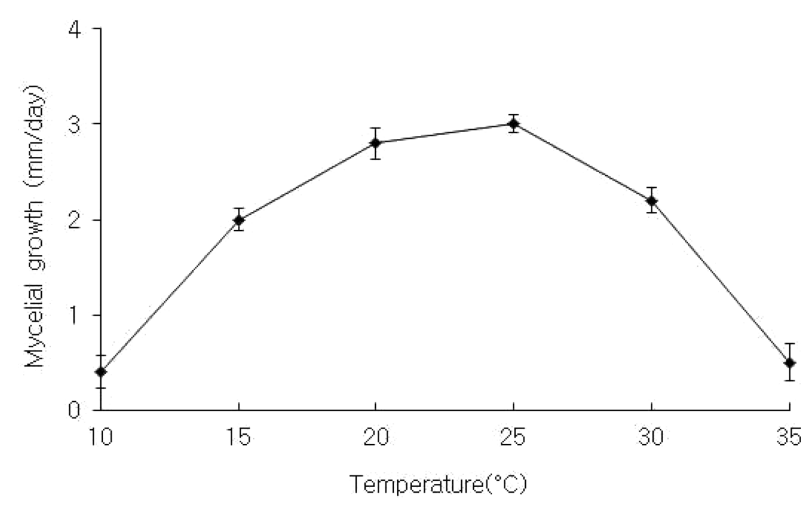

Fig. 2. Mycelial growth of $C$. militaris 'Yedang 3' on the PDA at different temperature. Bars indicate standard errors.

3 ' in the range of $8-9$ contradicting other report (Sung at al., 2002). These results suggest that $C$. militaris may have optimal $\mathrm{pH}$ range at $6 \sim 7$ in general but mycelial growth out of this range could be different with other strains.

The temperature favourable for the mycelial growth of $C$. militaris 'Yedang 3' was obtained at $20 \sim 25^{\circ} \mathrm{C}$ (Fig. 2). Similarly Sung et al. (2002) reported that the mycelial growth of $C$. militaris strains was optimal at $25^{\circ} \mathrm{C}$. Although the mycelial growth was favourable at the range of $20-25^{\circ} \mathrm{C}$, the fungus appeared to be suppressed at the high temperature of $35^{\circ} \mathrm{C}$. However, in the static liquid culture in Erlenmeyer flasks, optimum temperature for mycelial growth was $15-20^{\circ} \mathrm{C}$ and cordycepin production was the highest at $25^{\circ} \mathrm{C}$ (Hung et al., 2009). In the submerged culture, the optimal temperature for mycelial growth were found to be at

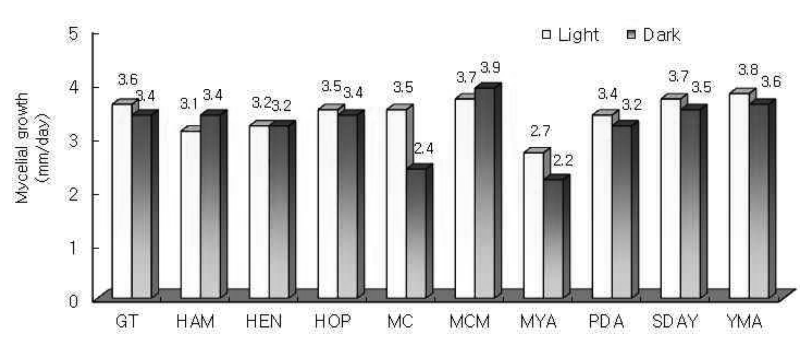

Fig. 3. Mycelial growth of $C$. militaris 'Yedang 3' on various culture media under light and dark conditions.

$20^{\circ} \mathrm{C}$ (Park et al., 2001). Therefore, the mycelial growth in C. militaris might be affected differently by strain and culture method.

Ten different culture media were used to test mycelial growth of $C$. militaris 'Yedang 3' under light and dark conditions (Fig. 3). Of ten culture media, MCM was the most favorable for mycelia growth followed by SDAY and YMA. When assessed twenty two different types of media and categorized into poor and rich media in nutritional sources, Shrestha et al. (2006) reported a similar result that SDAY, YMA and MCM developed abundant mycelial density. $\mathrm{He}$ suggested that nutritionally rich media produced abundant mycelial growth and this may be because of the sufficiency of all the requirements for the vegetative growth in C. militaris. After 15 days of mycelial culture under light condition, seven media from 10 culture media tested were found to be favorable for mycelial growth which were GT, HOP, MC, MYA, PDA, SDAY and YMA media. Mycelial growth in MC showed the most difference with $1.1 \mathrm{~mm}$ between light and dark conditions. Although the mycelial growth was greatest in MCM under dark condition, the fungus grew fastest in YMA under light condition. Light was found to be the most critical factor in determining mycelial growth characteristics such as the density, texture, and pigmentation (Shrestha et al., 2006). Similarly, light was regarded as one of most important environmental factors affecting fruiting body production because no fruiting body was formed in darkness in C. militaris (Sato and Shimazu, 2002). In general, optimum light intensity was in the range of 500-1,000 $\mathrm{lx}$ and the upper limit was 1,400 lx (Hong et al., 2010, 


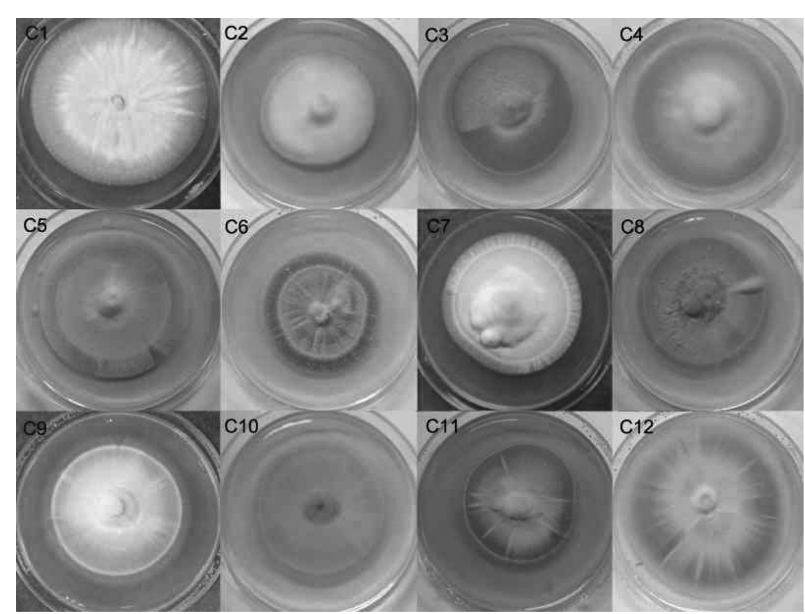

Fig. 4. Mycelial colonies of $C$. militaris 'Yedang 3' on various carbon sources.

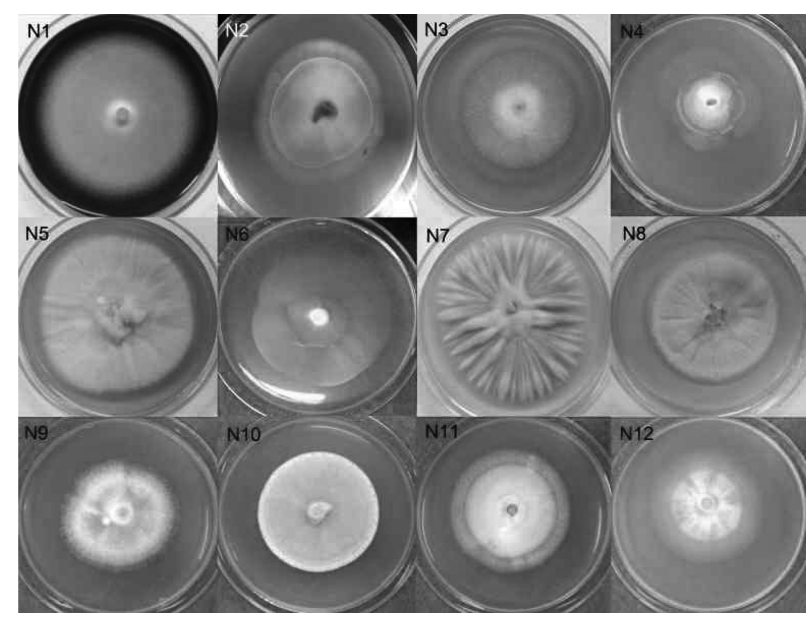

Fig. 5. Mycelial colonies of C. militaris 'Yedang 3' on various nitrogen sources.

Sato and Shimazu, 2002). It is apparent that light plays important role in mycelial growth and fruiting body formation but further studies are required in the subject of mycelial growth conditions in relation to fruiting body formation.

The mycelial colonies of C. militaris 'Yedang 3' were tested on various caron and nitrogen sources (Fig. 4 and 5). The morphological characteristics such as the mycelial growth, colonial color and shape were varied on different carbon and nitrogen sources. The colonial color by various carbon sources were white on $\mathrm{C} 1, \mathrm{C} 2, \mathrm{C} 7, \mathrm{C} 9$, yellowish on $\mathrm{C} 5$ and $\mathrm{C} 10$ and orange on C3, C4, C6, C11, $\mathrm{C} 12$. The colonial color by various nitrogen sources were white on N1, N3, N9, N10, N11, yellowish on $\mathrm{N} 4, \mathrm{~N} 5, \mathrm{~N} 12$, orange on $\mathrm{N} 7$ and N8. Also

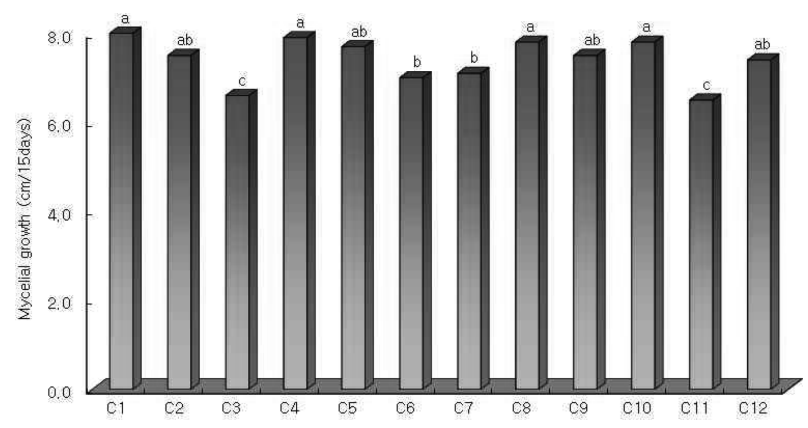

Fig. 6. Mycelial growth of C. militaris 'Yedang 3' on various carbon sources.

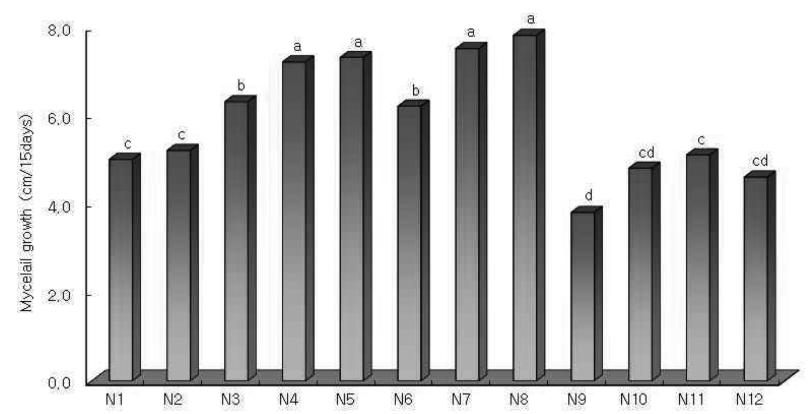

Fig. 7. Mycelial growth of C. militaris 'Yedang 3' on various nitrogen sources.

transparent colonies were examined on N2, and N6. Colonial shapes were different depending on carbon and nitrogen sources from cottony, streak, concentric to radial. Such morphological characteristics on different media by various carbon and nitrogen sources may be useful to characterize or give characteristic features in different strain.

The mycelial growth of C. militaris 'Yedang 3' varied significantly on various carbon sources but it was not strict on the requirement of carbon sources compared with nitrogen sources. $\mathrm{C} 1, \mathrm{C} 4, \mathrm{C} 8$ and C10 were the most suitable carbon sources for the mycelial growth followed by $\mathrm{C} 2, \mathrm{C} 5, \mathrm{C} 9$ and $\mathrm{C} 12$. The fungus grew slowly On C3 and C11 as carbon sources (Fig. 6).

After 15 days of culture, it was found that mycelial growth was affected significantly on various nitrogen sources. N8, N7, N5 and N5 was favorable for the mycelial growth of $C$. militaris 'Yedang 3' (Fig. 7). These were followed by N3, N6 and N11. The least mycelial growth was recorded on N9 nitrogen source. In the results, organic nitrogen sources such as N5, N7, N8 


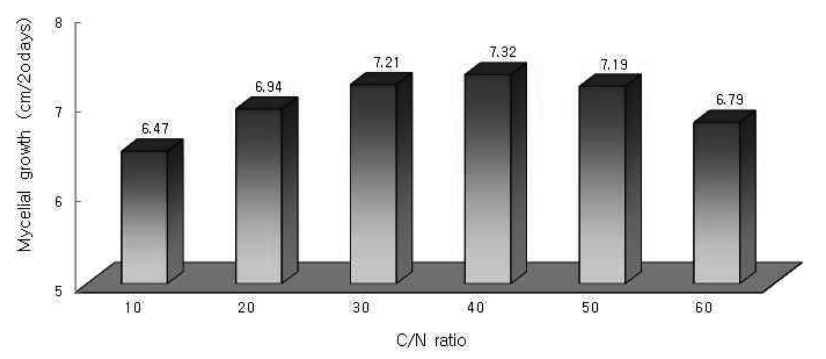

Fig. 8. Mycelial growth of C. militaris 'Yedang 3' on various $\mathrm{C}: \mathrm{N}$ ratio.

showed favorable mycelial growth compared with inorganic sources such as N1, N9, N10. Such results indicated that the organic nitrogen sources supported the mycelial growth of C. militaris 'Yedang 3' better than the inorganic sources, because the inorganic nitrogen sources are not easily utilized than organic sources.

Sucrose and peptone were suitable as carbon and nitrogen sources for the mycelial growth of $C$. militaris 'Yedang 3'. The $\mathrm{C}: \mathrm{N}$ ratio was adjusted to $10,20,30$, and 40 with varying quantities of sucrose as carbon source and peptone as nitrogen source. With increasing C:N ratio the mycelial growth was increased from 10:1 to 40:1 and the greatest mycelial growth was resulted at 40:1 (Fig. 8). At the high carbon ratio in the range of $50: 1$ to 60:1, the mycelial growth decreased from $7.19 \mathrm{~cm}$ to $6.79 \mathrm{~cm}$. Therefore it was observed that the optimum C:N ratio for mycelial growth was 30 40 range in C. militaris 'Yedang 3' as compared with other $\mathrm{C}: \mathrm{N}$ ratios. Different carbon, nitrogen and $\mathrm{C}: \mathrm{N}$ ratio with different strains have been investigated (Mao and Zhong, 2004; Mao et al., 2005; Shih et al., 2006) on mycelial growth of $C$. militaris but the direct comparison of the various studies is very difficult because the culture conditions, carbon and nitrogen sources used are different.

\section{References}

Che, Z. M., Wang, Y., Zhou, L. L., and Tang, C. L. 2004. Study on the breeding of a new variety of Cordyceps militaris by mutated with ultraviolet radiation. Food Ferment. Ind. 30 : 35-38.

Choi, I. Y., Choi, J. S., Lee, W. H., Yu, Y. J., Joung, G. T., Ju, I. O. and Choi, Y. K. 1999. The condition of production of artificial fruiting body of Cordyceps militaris. Kor. J. Mycol. 27 : 243-248.

Choi, Y. S., Kim, H. K., Lee, B. J., and Kim, Y. G. 2009. Characteristics and breeding of a new variety Cordyceps militaris 'Yedang 3'. J. Mushroom Sci. Production 7 : 182-186.

Das, S. K., Masuda, M., Sakurai, A., and Sakakibara, M. 2010. Medicinal uses of the mushroom Cordyceps militaris: Current state and prospects. Fitoterapia 81 : 961-968.

De Julian-Ortiz, J. V., Galvez, J., Munoz-Collado, C., Garcia-Domenech, R., and Gimeno-Cardona, C. 1999. Virtual combinatorial syntheses and computational screening of new potential anti-herpes compounds. J. Med. Chem. 42 : 3308-3314.

Dong, J. Z., Lei, C., Ai, X. R., and Wang, Y. 2012. Selenium enrichment on Cordyceps militaris Link and analysis on its main active components. Appl. Biochem. Biotechnol. 166 : 1215-1224.

Du, A. L., Zhang, X., and Zhang, H. Z. 2010. A new high cordycepin Cordyceps militaris cultivar 'Haizhou 1'. Acta Hortic. Sin. 37 : 1373-1374.

Fan, L., Pan, H., Scoccol, A. T., Pandey, A., and Soccol, C. R. 2006. Advances in mushroom research in the last decade. Food Technol. Biotechnol. 44 : 303-311.

Gao, X. H. 2008. Mating system of Cordyceps militaris. Acta Edulis Fungi 15 : 6-10.

Gui, Z. Z. and Zhu, Y. H. 2008. Advance on cultivation, bioactive compound and pharmacological mechanism of Cordyceps militaris. Sci. Seri 34 : 178-184.

Guo, H. P. and Yang, Z. M. 1999. Progress in research of pharmacological of Cordyceps sinensis. Traditional Herbal Drugs 30 : 231-233.

Hung, L. T., Keawsompong, S., Hanh, V. T., Sivichai, S., and Hywel-Jones, N. L. 2009. Effect of temperature on cordycepin production in Cordyceps militaris. Thai J. Agaric. Sci. 42 : 219-225.

Jiang, X. L. and Sun, Y. 1999. The determination of active components in various Cordyceps militaris strains. Acta Edulia Fungi 6 : 47-50.

Jiang, Y. and Yao, Y. J. 2003. Anamorphic fungi related to Cordyceps sinensis. Mycosystema 22 : 161-176.

Li, M. F. 2007. Molecular biology studies on the different phenotypes of fruiting-body forming of Cordyceps militaris. Master Thesis. Guizhou Univ., Guiyang, China.

Liang, Z. Q. 2001. current situation and ponderation of Cordyceps Fr. research and exploitation in China. Acta Edulis Fungi 8 : 53-62.

Liu, X. M., Cheng, Y. H., and Tian, D. 1999. Progress in the research of pharmacological Cordyceps in China. Natl. Product Res. Development 11 : 87-91.

Liu Z. Y. 1999. Studies on relationship between Cordyceps spp. and their anamorphs. $\mathrm{PhD}$ dissertation. Huazhong Agric. Univ., China.

Ma, T. Feng, Y., Wu, X. P., Zhang, Y. H., Ma, Y., and Wang, Z. L. 2007. Primary investigation of a host insect of Cordyceps militaris and analysis of its main ingredients. For. Res. 20 : 63-67. 
Mao, X. B., Eksriwong, T., Chauvatcharin, S., and Zhong, J. J. 2005. Optimization of carbon source and C:N ratio for cordycepin production by submerged cultivation of medicinal mushroom Cordyceps militaris. Process Biochem. 40 : 1667-1672.

Mao, X. B. and Zhong, J. J. 2004. Hyperproduction of cordycepin by two-stage dissolved oxygen control in submerged cultivation of medicinal mushroom Cordyceps militaris in bioreactors. Biotechnol. Prog. 20 : 1408-1413.

Mao, X. B. and Zhong, J. J. 2006. Significant effect of $\mathrm{NH}_{4}^{+}$on cordycepin production by submerged cultivation of medicinal mushroom Cordyceps militaris. Enzyme Microbiol. Technol. 38 : 343-350.

Masuda, M., Urabe, E., Honda, H., Sakurai, A., and Sakakibara, M. 2007. Enhanced production of cordycepin by surface culture using the medicinal mushroom Cordyceps militaris. Enzyme Microbiol. Technol. 40 : 1199-1205.

Oh, S. W., Kim, S. H., Song, H. N., and Han, D. S. 2003. Comparative chemical compositions of four kinds of Tochkaso. Kor. J. Food Sci. Technol. 35 : 15-22.

Park, J. P., Kim, S. W., Hwang, H. J., and Yun, J. W. 2001. Optimization of submerged culture conditions for the mycelial growth and exo-biopolymer production by Cordyceps militaris. Lett. Appl. Microbiol. 33 : 76-81.

Patel, K. J. and Ingalhalli, R. S. 2013. Cordyceps militaris (L.: Fr.) Link An important medicinal mushroom. J. Pharm. Phytochem. 2 : 315-319.

Sato, H. and Shimazu, M. 2002. Stroma production for Cordyceps militaris (Clavicipitales: Clavicipitaceae) by injection of hyphal bodies to alternative host insects. Appl. Entomol. Zool. 37 : 85-92.

Shih, I. L., Tsai, K. L., and Hsieh, C. Y. 2006. Effects of culture conditions on the mycelial growth and bioactive metabolite production in submerged culture of Cordyceps militaris. Biochem. Eng. J. 33 : 193-201.

Shrestha, B., Lee, W. H., Han, S. K, and Sung, J. M. 2006. Observations on some of the mycelial growth and pigmentation characteristics of Cordyceps militaris isolates. Mycobiol. 34 : 83-91.

Stensrud, Ø, Hywel-Jones, N. L., and Schumacher, T. 2005. Towards a phylogenetic classification of Cordyceps: ITS nrDNA sequence data confirm divergent lineages and paraphyly. Mycol. Res. 109 : 41-56.

Sun, J. D., Xiong, S. T., and Wang, P. 2009. Study on biological and cultivated characters of Cordyceps militaris SN3. J. Fungal Res. 7 : 148-152.

Sung, G. H., Hywel-Jones, N. L., Sung, J. M. Luangsa-Ard, J. J., Shrestha, B., and Spatafora, J. W. 2007. Phylogenetic classification of Cordyceps and the clavicipitaceous fungi. Stud. Mycol. 57 : 50-59.

Sung, J. M., Choi, Y. S., Shrestha, B., and Park, Y. J. 2002. Investigation on artificial fruiting of Cordyceps militaris. Kor. J. Mycol. 30 : 6-10.

Sung, J. M., Park, Y. J., Lee, J. O., Han, S. K., Lee, W. H., Choi, S. K., and Shrestha, B. 2006. Effect of preservation periods and subcultures on fruiting body formation of Cordyceps militaris in vitro. Mycobiol. 34 : 196-199.
Tan, Q., Cai, T., Wei, J., Feng, A. P., Mao, W. J., and Bao, D. P. 2011. Molecular identification of mating type genes in asexual spores of Cordyceps militaris. ICMBMP7 2011 : 52-56.

Tong, Y. K., Kuang, T., Wu, Y. X., Zhang, Q. Y., and Ren, J. 1997. Comparison of components of Cordyceps mycelium and natural Cordyceps sinensis. Shi Pin Yan Jiu Yu Kai Fa 18 : 40-42.

Turgeon, B. G., and Yoder, O. C. 2000. Proposed nomenclature for mating type genes of filamentous ascomycetes. Fungal Genet. Biol. 31 : 1-5.

Wang, L, Zhang, W. M., Hu, B., Chen, Y. Q., and Qu, L. H. 2008. Genetic variation of Cordyceps militaris and its allies based on phylogenetic analysis of rDNA ITS sequence data. Fungal Diversity. 31 : 147-155.

Wang, X. Y., Rong, Y. W., Xu, L., Zhang, N. S., Li, C. R., and Fan, M. Z. 2010. Electrophoretic karyotype analysis of Paecilomyces militaris, the anamorph of Cordyceps militaris. J. Anhui Agric. Univ. 37 : 716-719.

Wen, T. C., Li, M. F., Kang, J. C., and He, J. 2012. A molecular genetic study on fruiting-body formation of Cordyceps militaris. Afr. J. Microbiol. Res. 6 : 52155221.

Wong, Y. Y., Moon, A., Duffin, R., Barthet-Barateig, A., Meijer, H. A., Clemens, M. J., and De Moor, C. H. 2010. Cordycepin inhibits protein synthesis and cell adhesion through effects on signal transduction. J. Biol. Chem. 285 : 2610-2621.

Wu, F. Y., Yan, H., Ma, X. A., Jia, J. Q., Zhang, G. Z., Guo, X. J., and Gui, Z. Z. 2011. Structural characterization and antioxidant activity of purified polysaccharide from cultured Cordyceps militaris. Afr. J. Microbiol. Res. 5 : 2743-2751.

Yokoyama, E., Yamagishi, K., and Hara, A. 2004. Development of a PCR-based mating-type assay for Clavicipitaceae. FEMS Microbiol. Lett. 237 : 205-212.

Yokoyama, E., Yamagishi, K., and Hara, A. 2003. Structures of the mating-type loci of Cordyceps takaomontana. Appl. Environ. Microbiol. 69 : 5019-5022.

Yoo, H. S., Shin, J. W., Cho, J. H., Son, C. G., Lee, Y. W., Park, S. Y., and Cho, C. K. 2004. Effects of Cordyceps militaris extract on angiogenesis and tumor growth. Acta Pharm. Sinica 25 : 657-665.

Yu, H. M., Wang, B. S., Huang, S. C., and Duh, P. D. 2006. Comparison of preventive effects between cultured Cordyceps militaris and natural Cordyceps sinensis against oxidative damage. J. Agaric. Food Chem. 54 : 3132-3138.

Zheng, P., Xia, Y. L., Xiao, G. H., Xiong, C. H., Hu, X., Zhang, S. W., Zheng, H. J., Huang, Y., Zhou, Y., Wang, S. Y., Zhao, G. P., Liu, X. Z., St Leger, R. J., and Wang, C. S. 2011. Genome sequence of the insect pathogenic fungus Cordyceps militaris, a valued traditional Chinese medicine. Genome Biol. 12 : R116

Zhou, X. W., Gong, Z. H., Su, Y., Lin, J., and Tang, K. X. 2009. Cordycepin fungi: natural products, pharmacological functions and developmental products. J. Pharm. Pharmacol. 61 : 279-291. 